\title{
Analysis of a Low-Invasive Method to Create Full- Thickness Articular Cartilage Defects in a Rat Model
}

\author{
IKUFUMI TAKAHASHI, RPT, MS ${ }^{1,2)}$, MASAHIRO HOSO, MD, $\mathrm{PhD}^{2)}$, \\ TARO MATSUZAKI, RPT, MS ${ }^{2}$ \\ 1) Department of Rehabilitation, Houju Memorial Hospital: 11-71 Midorigaoka, Nomi City, Ishikawa, \\ 923-1226 Japan.TEL:+81761-51-5551,E-mail:reha-pt@houju.jp \\ ${ }^{2)}$ Division of Health Sciences, Graduate School of Medical Science, Kanazawa University
}

\begin{abstract}
Purpose] We investigated a rat model of full-thickness articular cartilage defect without joint open surgery and histopathologically analyzed the time course of the tissue repair. [Subjects] A total of 40 male Wistar rats aged 9 weeks were used. [Method] Full-thickness defects of the articular cartilage were created over the capsule at the loading portion in the medial condyle of the femur. Ten rats each were randomly allocated to 4 groups, which were evaluated at $0,4,8$ and 12 weeks after surgery. At the end of each period, knee joints were examined histopathologically. [Results] Full-thickness articular cartilage defects were created uniformly in our model. The defects were spontaneously resurfaced with hyaline-like tissue at 4 weeks postoperatively, but some articular cartilage remnants with aseptic necrosis and cloning of chondrocytes were found in the repair tissue. From 8 weeks to 12 weeks after surgery, the hyaline tissue was maintained, and the aseptic necrosis and cloning were observed in the partially repair tissue. [Conclusion] We have developed a rat model producing identically sized full-thickness defects of the articular cartilage.

Key words: Articular cartilage, Full-thickness defect, Rat
\end{abstract}

(This article was submitted Jun. 10, 2011, and was accepted Jul. 12, 2011)

\section{INTRODUCTION}

Traumatic articular cartilage defects of the knee are a common cause of pain and functional disability in orthopedics and sports medicine ${ }^{1)}$. Unfortunately, when cartilage is damaged due to injury or disease, it has a limited capacity to heal. Human adult articular cartilage contains no blood supply, neural network, or lymphatic drainage $^{2,3)}$. Furthermore, the abilities of repair and division of chondrocytes are limited, and nutrition supplied to the cartilage is exclusively provided by synovial fluid produced by the synovial membrane. As a result, full-thickness articular cartilage defects that penetrate through the cartilage undergo regenerative repair of hyaline cartilage under restricted conditions ${ }^{4)}$. Many researchers have studied the mechanism of articular cartilage regeneration. However it has not been fully clarified and there is still no consensus opinion about the tissue repair process ${ }^{5-7)}$. In addition, the gene expression, the cell differentiation and the growth factors that are needed for the cartilage regeneration remain uncertain ${ }^{8)}$.

Joint motion and synovial fluid are important for transporting chemical mediators to repair tissue, promoting chondrogenesis and maintaining cartilage phenotype ${ }^{9}$. Many previous studies have used animal models in which the articular capsule is incised and full-thickness articular cartilage defects are created with the joint surgically open.
However, cartilage dissection, synovial membrane injury and loss of synovial fluid accompany the joint opening. The nutrition supplied to the cartilage depends on synovial fluid; therefore, these accompanying factors may have an unfavorable influence on cartilage repair.

In present study, we described a rat model of fullthickness articular cartilage defects without joint opening. The purposes of this study were to investigate this model and to histopathologically analyze the time course of the tissue repair.

\section{SUBJECTS AND METHODS}

This investigation was approved by the Animal Research Committee of Kanazawa University Graduate school of Medicine, Kanazawa, Japan (approval No.101763). All procedures for animal care and treatment were performed in accordance with the Guidelines for the Care and Use of Laboratory Animals at Kanazawa University. Forty 9-weekold Wistar male rats were used in this study. Ten rats each were randomly allocated to one of 4 groups, which were evaluated at 0 (immediately after surgery), 4, 8 and 12 weeks after surgery. The animals were kept under normal conditions for one week before the start of the experiments in order to acclimatize them to the environment. They were housed, 2 per a cage, in a room maintained under a 12-hour light-dark cycle, and food and water were given ad libitum. 


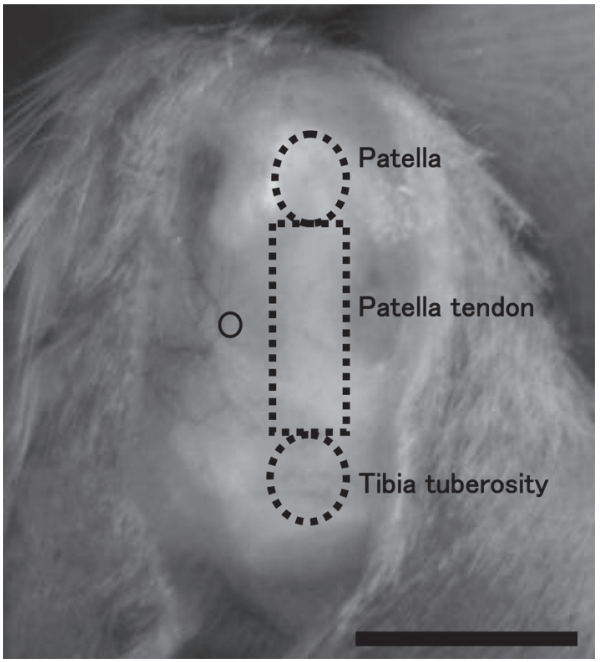

Fig. 1. The position of the full-thickness articular cartilage defect over the capsule.

The defect was created at the inside position of the inner margin of the tendon at the height of the center of the patella tendon (circle). Scale bar $=$ $10 \mathrm{~mm}$.

The rats were anesthetized by an intraperitoneal injection of sodium pentobarbital at a dose of $40 \mathrm{mg} / \mathrm{kg}$. After shaving the left knees, they were disinfected and a parapatellar incision was performed to expose the knee joint. In maximum flexion of the knee, full-thickness defects $(0.8 \mathrm{~mm}$ diameter, $2.0 \mathrm{~mm}$ depth $)$ of articular cartilage were created over the capsule by a Kirschner wire $(0.8 \mathrm{~mm}$ in diameter) in the medial condyle (Fig. 1). At the height of the center of the patella tendon, the defect was created at the medial position of the inner margin of the tendon (half tendon width of distance). The wire was marked at position $2.0 \mathrm{~mm}$ from the tip to ensure invasive depth uniformity. After the creation of the defect, the skin was sutured. All rats were allowed to move freely in their cages without knee immobilization after surgery.

At $0,4,8$ and 12 weeks after surgery, rats were sacrificed by an intraperitoneal injection of an overdose of sodium pentobarbital. Immediately after death, both hindlimbs were disarticulated at the hip joint. All left knees were fixed in $10 \%$ neutral buffered formalin for 72 hours, decalcified with Decalcifying Solution A (Plank Rychlo Method, Wako Pure Chemical Industries, Ltd., Osaka, Japan) for 72 hours. The knees were excised, deacidified in 5\% sodium sulfate solution for 72 hours, dehydrated in an ethanol after water washing and embedded in paraffin wax. Saggital sections (3 $\mu \mathrm{m})$ were stained with hematoxylin and eosin, and examined and imaged using a light microscope and digital camera (BX-51 and DP-50; Olympus, Co., Tokyo, Japan).

The extent of cartilage regeneration was studied on the digital image and the width and depth of the defects were measured at 0 and 4 weeks after surgery using image processing software (Image $\mathrm{J}$ for Windows version 1.38).
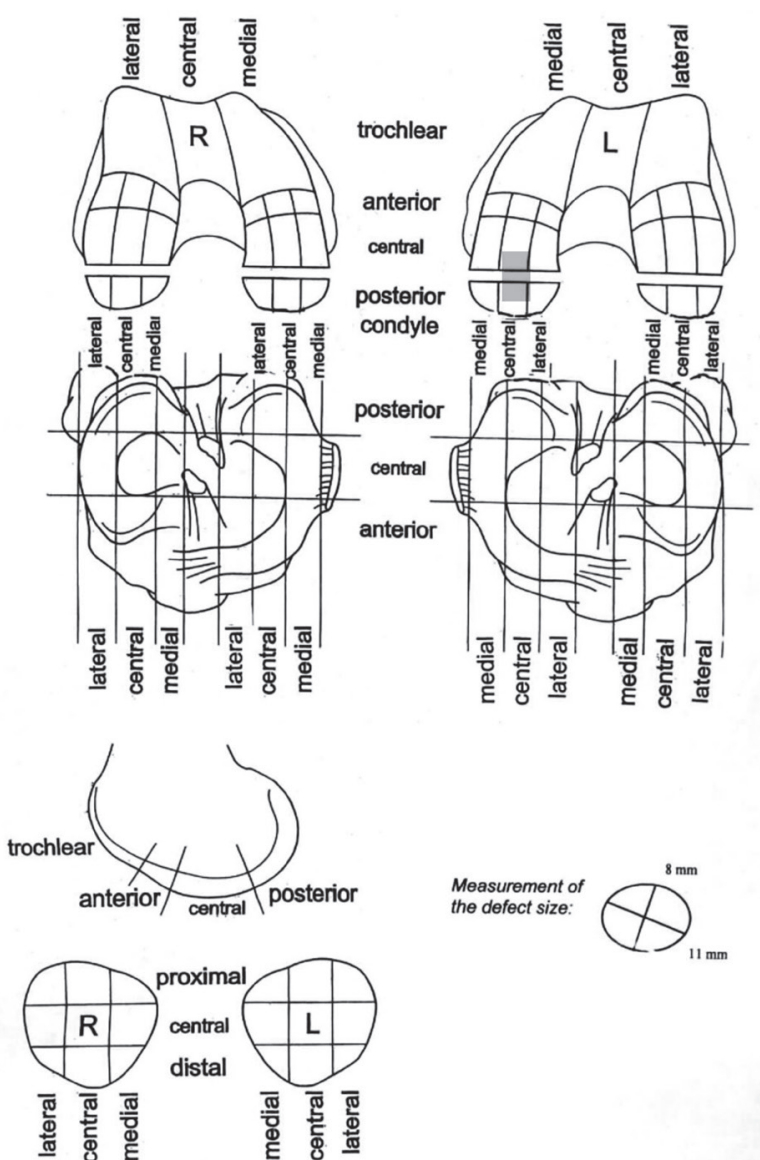

Fig. 2. The International Cartilage Repair Society Knee Lesion Mapping System.

The position of the defect was evaluated macroscopically with this system. All defects were located in the central area in the frontal and saggital planes (gray area).

Comparisons of the width and depth of the defects at 0 and 4 weeks were conducted with the unpaired Student's t-test. In all statistical analyses, a value of $\mathrm{p}<0.05$ was considered statistically significant.

To evaluate the reproducibility of the defect, fullthickness defects were created post mortem in all right knees and compared after joint opening. The capsules of the knees were cut immediately, and the joints were opened. After total resection of bilateral menisci and cruciate ligaments of knee, the position of the defect was studied macroscopically with The International Cartilage Repair Society (ICRS) Knee Lesion Mapping System (Fig. 2).

\section{RESULTS}

All animals were conscious and started to walk within several hours after surgery. No rat showed signs of knee infection or swelling, or died during the experimental period. Thus, the inflammation was macroscopically and microscopically well-controlled. 

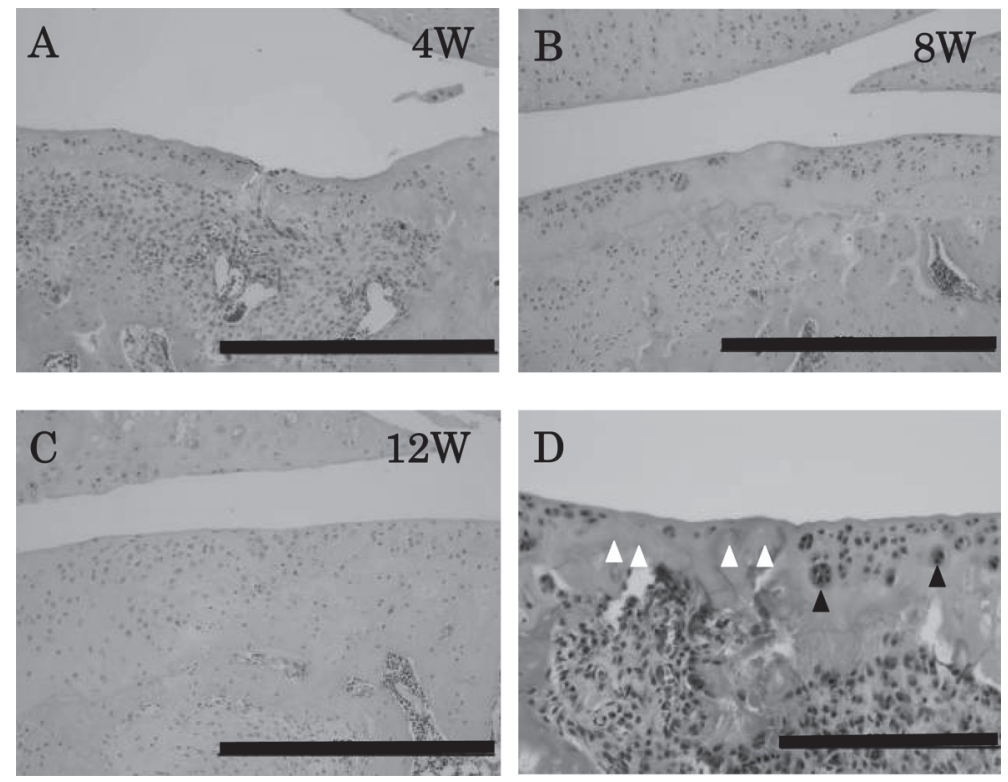

Fig. 3. Histological findings of the repair process. Saggital sections were stained with hematoxylin and eosin at 4 weeks (A), 8 weeks (B, D), and 12 weeks (C).

The defects were spontaneously resurfaced with hyaline-like tissue from 4 to 12 weeks. Aseptic necrosis (white arrow head) and cloning of chondrocytes (black arrow head) were observed in the repair tissue from 4 to 12 weeks postoperatively (D). (A-C) scale bar $=500 \mu \mathrm{m}$, original magnification $\times 100$; (D) scale bar $=200 \mu$ m, original magnification $\times 200$.

All defects were located in the central area in the frontal and saggital planes according to the ICRS Knee Lesion Mapping System.

For the measurement of the defects, the mean \pm SD values for the width of the defects at 0 and 4 weeks were $704.7 \pm 95.1 \mu \mathrm{m}$ and $760.3 \pm 71.3 \mu \mathrm{m}$, respectively. The values for the depth at 0 and 4 weeks were $1948.6 \pm 311.5$ $\mu \mathrm{m}$ and $1799.9 \pm 302.2 \mu \mathrm{m}$, respectively. In the comparison of the extent of defects at 0 and 4 weeks, there were no significant differences in the width or depth of the defects.

In the histological examination, at 4 weeks after surgery, the defects spontaneously resurfaced with hyaline-like tissue, but the surfaces were irregular. Some remnants of articular cartilage were comprised in the repair tissues (Fig. $3)$. The remnants regenerated concomitantly with aseptic necrosis and cloning of chondrocytes. From 8 weeks to 12 weeks after surgery, the hyaline tissue, which is similar to normal articular cartilage, was maintained, and the aseptic necrosis and cloning were observed in the partially repair tissue.

\section{DISCUSSION}

In many studies ${ }^{4-7,10)}$, a medial parapatellar skin incision followed by soft tissue dissection has been used to expose the capsule. The capsule is incised and the patella is dislocated laterally to expose the femur. After creating the defects, the patella is repositioned, and the capsule and skin are closed independently with nylon sutures. This method is considered to have a high degree of accuracy and reliability for the macroscopic confirmation of the defect location. Nevertheless, joint opening is accompanied by cartilage desiccation, synovial membrane injury, loss of synovial fluid and decrease of cartilage nutrition. Therefore, these accompanying factors may have unfavorable influences on cartilage repair.

In the present study, we described a novel rat model for full-thickness articular cartilage defects without joint opening to reduce the degree of invasion in the knee joint. Using this method, we could easily create full-thickness articular cartilage defects with low invasion. It was not necessary to dislocate the patella and dissect any periarticular muscle. However, our method has consistency problems in the site and size of the defect because we cannot confirm the site macroscopically until animal sacrifice. We tried to standardize the depth of the defect by calculating semi-quantitatively the position of the defect over the capsule. The measurements of the defects showed that full-thickness defects of the articular cartilage were created at the same position with a high degree of accuracy and reliability. The position of the defect has been reported to be the loading area of the articulation ${ }^{10)}$. Arthritis and synovitis caused by infection and intra-articular bleeding were not observed in our low-invasive model. Therefore, side effects of surgery were not apparent during the study.

Histological examination revealed that the hyaline tissue, 
which is similar to normal articular cartilage, was maintained at 8 weeks postoperatively. Generally, fullthickness articular cartilage defects are believed to induce a fibrin clot formation in the area of the chondral defect ${ }^{3,11,12)}$. This clot contains pluripotent marrow-derived mesenchymal stem cells. These cells are able to differentiate into fibrocytes and chondrocytes, resulting in hyaline cartilage or fibrocartilage repair with varying amounts of type I, II, and X collagen content ${ }^{3,11,12)}$. However, there is no consensus over which cartilage is generated; hyaline cartilage or fibrocartilage ${ }^{5-7}$. It is known that cartilage repair is influenced by species and age, and by position, depth and size of the defect ${ }^{2,13,14)}$. It has been reported that defects are postoperatively repaired with hyaline cartilage from 8 to 12 weeks in small mammal like rabbits and rats.

Some remnants of articular cartilage were found in the repair tissue. The remnants were regenerated concomitantly with aseptic necrosis and cloning of chondrocytes. In previous studies, the remnants were removed by surgical equipment ${ }^{4,6,7,9,10)}$. In contrast, in the present study, we assume that the remnants were residues arising from the creation of the cartilage defects which remained in the repair tissue because most aseptic necrosis of chondrocytes was observed in the remnants. Cloning of chondrocytes in the remnants is observed as interstitial growth which is one of growth processes of cartilage. Cloning is a phase of cell division, and an activated state of metabolism. Mosaicplasty and autologous chondrocyte implantation are commonly performed as treatments to promote cartilage repair with normal cartilage tissue. Accordingly, in this study, one possible reason for the regeneration of hyaline cartilage might have been the remnants of the cartilage in the repair tissue. However, the degree of aseptic necrosis and cloning of chondrocytes remaining or produced was unclear. To clarify this issue, it will be necessary to evaluate not only the center, but the other parts of the repair tissue as well.

In conclusion, the present results suggest that the lowinvasive method described in this study is useful for creating full-thickness articular cartilage defects. Further study including the evaluation of all parts of the repair tissue should be conducted because the mechanical influence of joint motion and weight-bearing on cartilage repair is unknown.

\section{REFERENCES}

1) Reinold MM, Wilk KE, Macrina LC, et al.: Current concepts in the rehabilitation following articular cartilage repair procedures in the knee. $\mathrm{J}$ Orthop Sports Phys Ther, 2006, 36: 774-794.

2) Reinholz RR, Lu L, Yaszemski MJ, et al.: Animal models for cartilage reconstruction. Biomaterials, 2004, 25: 1511-1521.

3) Mankin HJ: Response of articular cartilage to mechanical injury. J Bone Joint Surg Am, 1982, 64: 460-466.

4) Anraku Y, Mizuta H, Sei A, et al.: Analyses of early events during chondrogenic repair in rat full-thickness articular cartilage defects. J Bone Miner Metab, 2009, 27: 272-286.

5) Gill TJ, Asnis PD, Berkson EM, et al.: Chondral defect repair after the microfracture procedure. Am J Sports Med, 2005, 33: 680-685.

6) Frisbie DD, Julia T, Trotter GW, et al.: Early events in cartilage repair after subchondral bone microfracture. Clin Orthop Relat Res, 2003, 407: 215227.

7) Shapiro F, Koide S, Glimcher MJ, et al.: Cell origin and differentiation in the repair full-thickness defects of articular cartilage. J Bone Joint Surg Am, 1993, 75: 532-553.

8) Ikeda T, Kamekura S, Mabuchi A, et al.: Combination of Sox5, Sox6, and Sox 9 provide signals sufficient for chondrogenesis. Arthritis Rheum, 2004, 50: $3561-3573$

9) Furukawa T, Eyre DR, Koide S, et al.: Biochemical studies on repair cartilage resurfacing experimental defects in the rabbit knee. J Bone Joint Surg Am, 1980, 62: 79-89.

10) Frisbie DD, Oxford JT, Trotter GW, et al.: Early events in cartilage repair after subchondral bone microfracture. Clin Orthop Relat Res, 2003, 407: 215-227.

11) Gill TJ, Asnis PD, Berkson EM, et al.: The treatment of articular cartilage defects using the microfracture technique. J Orthop Sports Phys Ther, 2006, 36: 728-738.

12) Williams RJ 3rd, Harnly HW: Microfracture: indications, technique, and results. Instr Course Lect, 2007, 56: 419-428.

13) Messenner K, Gillquist J: Cartilage repair. Acta Orthop Scand, 1996, 67: 523-529.

14) Yoshida M, Kubo T, Richard DC, et al.: Differences in the repair process of longitudinal and transverse injuries of cartilage in the rat knee. Osteoarthritis Cartilage, 1998, 6: 66-75. 